\title{
Clinicopathological features of programmed death ligand 1 expression with tumor-infiltrating lymphocyte, mismatch repair, and Epstein-Barr virus status in a large cohort of gastric cancer patients
}

\author{
Akihito Kawazoe ${ }^{1,2,3} \cdot$ Takeshi Kuwata $^{1,5}$ - Yasutoshi Kuboki ${ }^{2}$ Kohei Shitara ${ }^{2}$. \\ Akiko Kawano Nagatsuma ${ }^{1}$ Masaaki Aizawa ${ }^{4}$ Takayuki Yoshino ${ }^{2} \cdot$ \\ Toshihiko Doi $^{2}$ - Atsushi Ohtsu ${ }^{2,3}$ - Atsushi Ochiai ${ }^{1}$ (D) \\ Received: 7 May 2016/ Accepted: 2 August 2016/Published online: 14 September 2016 \\ (c) The International Gastric Cancer Association and The Japanese Gastric Cancer Association 2016
}

\begin{abstract}
Background Antibodies against programmed death 1 (PD1 ) and its ligand programmed death ligand 1 (PD-L1) have recently demonstrated promising results in gastric cancer (GC). PD-L1 expression, the presence of tumor-infiltrating lymphocytes (TILs), and mismatch repair (MMR) deficiency have been proposed as predictive biomarkers for anti-PD-1/PD-L1 antibodies. The aim of this study was to investigate the clinical relevance of PD-L1 expression with TIL, MMR, and Epstein-Barr virus (EBV) status in GC. Methods We performed a tissue microarray analysis in 487 advanced GC patients who underwent gastrectomy. PD-L1 expression on tumor cells (TCs) and tumor-infiltrating immune cells (TIICs), the densities of TILs, and MMR status were evaluated by immunohistochemistry. EBV was detected by in situ hybridization.
\end{abstract}

Electronic supplementary material The online version of this article (doi:10.1007/s10120-016-0631-3) contains supplementary material, which is available to authorized users.

Atsushi Ochiai

aochiai@east.ncc.go.jp

1 Division of Pathology, Research Cancer for Innovative Oncology, National Cancer Center Hospital East, 6-5-1 Kashiwanoha, Kashiwa, Chiba 277-8577, Japan

2 Department of Gastroenterology and Gastrointestinal Oncology, National Cancer Center Hospital East, Kashiwa, Japan

3 Juntendo University Graduate School of Medicine, Bunkyoku, Tokyo, Japan

4 Department of Surgery, Niigata Cancer Center Hospital, Niigata, Niigata, Japan

5 Department of Pathology and Clinical Laboratories, National Cancer Center Hospital East, Kashiwa, Japan
Results PD-L1 expression on TCs and TIICs, MMR deficiency, and EBV positivity were identified in 22.8, 61.4, 5.1 , and $5.1 \%$ cases respectively. PD-L1 expression was more frequently observed in the elderly (TCs $P=0.002$ ), in males (TCs $P=0.029$; TIICs $P=0.043$ ), in patients with poorly differentiated adenocarcinoma with solid-type histological features (TCs $P<0.001$; TIICs $P<0.001$ ), in patients with MMR deficiency (TCs $P<0.001$; TIICs $P<0.001$ ), and in patients with EBV positivity (TCs $P=0.001$; TIICs $P=0.050$ ). Strong association was observed between PD-L1 expression and high densities of CD3-positive, CD8-positive, or forkhead box P3 positive TILs (TCs $P<0.001$; TIICs $P<0.001$ ). Neither PD-L1 expression on TCs nor that on TIICs was an independent prognostic factor in multivariate analysis.

Conclusions In GC, PD-L1 expression was associated with distinct clinicopathological features, including high densities of TILs, MMR deficiency, and EBV positivity, but was not a prognostic factor.

Keywords Gastric cancer - Programmed death ligand 1 . Tumor-infiltrating lymphocytes - Mismatch repair status · Epstein-Barr virus

\section{Introduction}

Blockade of immune checkpoint molecules with monoclonal antibodies has recently emerged as a promising strategy for treating several malignancies [1-4]. Programmed death 1 (PD-1) belongs to the CD28 family of proteins and functions as a negative costimulatory receptor expressed on the surface of activated $T$ cells [5]. The binding of PD-1 and its ligands-programmed death ligand 1 (PD-L1) and programmed death ligand 2 (PD- 
L2)—on tumor cells (TCs) or immune cells can inhibit cytotoxic T-cell responses, allowing TCs to evade immune detection [5]. Accordingly, it has been considered that a blockade of this interaction restores the antitumor activity of T cells [5]. Clinical trials of anti-PD-1/PD-L1 antibodies have shown high response rates and significantly longer overall survival (OS) in several malignancies [1-4]. On the basis of these trials, the US Food and Drug Administration has approved two anti-PD-1 antibodies-nivolumab and pembrolizumab-for treatment of patients with advanced melanoma, non-small-cell lung cancer, and renal cell carcinoma. Pembrolizumab has also shown promising results in a phase I trial for patients with advanced gastric cancer (GC) [6]. Several phase III trials of anti-PD-1/PD-L1 antibodies are ongoing for the treatment of advanced GC.

Although there are no established biomarkers of antiPD-1/PD-L1 antibodies, some reports have shown that PDL1 expression on the membranes of TCs or tumor-infiltrating immune cells (TIICs) and tumor-infiltrating lymphocytes (TILs) was associated with better clinical outcomes following treatment with anti-PD-1/PD-L1 antibodies in several malignancies $[1,3,7,8]$. For instance, PD-L1 expression in non-small-cell lung cancer and preexisting $\mathrm{CD}^{+}$TILs in melanoma have been correlated with the greater efficacy of pembrolizumab [3, 8]. In GC, there was also some correlation between PD-L1 expression on TCs or TIICs and the tumor response to pembrolizumab in a phase I trial [6]. The Cancer Genome Atlas Research Network [9] reported that amplification of the $C D 274$ gene (which encodes PD-L1) and the PDCDILG2 gene (which encodes PD-L2) was frequently observed in Epstein-Barr virus (EBV)-positive GC. Moreover, a recent clinical trial reported that mismatch repair (MMR) status predicted a clinical benefit for treatment with pembrolizumab [10]. However, information regarding the clinical relevance of PD-L1 expression with TIL, MMR, and EBV status in GC has been limited.

In this study, we investigated the clinicopathological features of PD-L1 expression on TCs and TIICs, as well as TIL, MMR, and EBV status in a large cohort of stage III and stage IV GC patients using tissue microarrays (TMAs).

\section{Materials and methods}

\section{Patients and TMAs}

Formalin-fixed, paraffin-embedded tumor samples from 487 patients with stage III or stage IV GC who underwent gastrectomy at our hospital from January 2002 to December 2010 were examined. None of the patients underwent systematic chemotherapy before surgery. The construction of the TMAs with these tumors has been described elsewhere [11]. Briefly, two representative tumor cores ( $2 \mathrm{~mm}$ in diameter) mostly located in the invasive area of each tumor were obtained from the same formalin-fixed, paraffin-embedded tissue block in each case. Serial $4-\mu \mathrm{m}$ sections were prepared and used for hematoxylin and eosin staining, immunohistochemistry (IHC), and in situ hybridization (ISH). Clinicopathological characteristics, including age, sex, tumor location, histological type, lymphatic invasion, venous invasion, depth of invasion, lymph node metastasis, presence of residual tumor on the resection margin, and adjuvant chemotherapy, were reviewed from medical records. The disease stage was classified according to the TNM criteria of the Union for International Cancer Control (seventh edition). Histological types were classified according to the Japanese classification of gastric carcinoma (third English edition). The study protocol was approved by the Institutional Review Board at the National Cancer Center.

\section{Immunohistochemistry}

The primary antibodies used for IHC were anti-PD-L1 (SP142) rabbit monoclonal antibody, CONFIRM anti-CD3 (2GV6) rabbit monoclonal antibody, CONFIRM anti-CD4 (SP35) rabbit monoclonal antibody, anti-CD8 (C8/144B) mouse monoclonal antibody, anti-forkhead box P3 (FOXP3; 236A/E7) mouse monoclonal antibody, antimutL homolog 1 (MLH1; ES05) mouse monoclonal antibody, anti-mutS homolog 2 (MSH2; FE11) mouse monoclonal antibody, anti-postmeiotic segregation increased 2 (PMS2; EP51) rabbit monoclonal antibody, and anti-mutS homolog 6 (MSH6; EP49) rabbit monoclonal antibody. Antibodies against PD-L1, CD3, and CD4 were purchased from Ventana (Tucson, AZ, USA), the antibody against FOXP3 was from Abcam (Cambridge, MA, USA), and all the other antibodies were from Dako (Copenhagen, Denmark). CD3, CD4, and CD8 IHC was performed with a BenchMark ULTRA fully automated slide processing system (Ventana) according to the manufacturer's instructions. MLH1, MSH2, PMS2, and MSH6 IHC was performed with a Dako autostainer according to the manufacturer's instructions. For PD-L1 and FOXP3 staining, the sections were deparaffinized in xylene, dehydrated with graded ethanol, and then immersed in methanol with $0.3 \%$ hydrogen peroxidase for $20 \mathrm{~min}$ to block endogenous peroxidase. Antigen retrieval was performed at $121^{\circ} \mathrm{C}$ for $10 \mathrm{~min}$ in a pressure cooker (Pascal pressurized heating chamber) followed by placement of sections in Dako target retrieval solution for PD-L1 or citrate buffer for FOXP3. The sections were immersed in $2 \%$ normal swine serum in phosphate-buffered saline to block nonspecific binding for $30 \mathrm{~min}$ at room temperature. The slides were then incubated overnight at $4{ }^{\circ} \mathrm{C}$ with the primary antibody. After 
they had been washed five times with phosphate-buffered saline, the slides were incubated with labeled polymer horseradish peroxidase rabbit/mouse antibody for $30 \mathrm{~min}$ (Envision Plus detection system; Dako). After extensive washing with phosphate-buffered saline, the color reaction was developed in $2 \% 3,3^{\prime}$-diaminobenzidine in $50 \mathrm{mmol} /$ $\mathrm{L}$ tris(hydroxymethyl)aminomethane buffer (pH7.6) containing $0.3 \%$ hydrogen peroxide for $4 \mathrm{~min}$. Background staining was performed with Mayer's hematoxylin solution, and sections were then dehydrated through ascending alcohols to xylene and mounted on slides.

\section{Evaluation of PD-L1 expression}

All tissue cores immunohistochemically stained with the anti-PD-L1 antibody were evaluated by two pathologists (A.K. and T.K.). Specimens were scored on the basis of the percentage of stained TCs or TIICs by IHC: 0 , less than $1 \% ; 1+$, from $1 \%$ to less than $10 \% ; 2+$, from $10 \%$ to less than $20 \% ; 3+, 20 \%$ or more (Fig. 1). PD-L1-positive cases on TCs or TIICs were defined by the presence of at least $1 \%$ of TCs or TIICs with membrane staining. A higher score was selected if two cores from the same case exhibited different PD-L1 expression scores.

\section{Measurement of TILs}

Stained slides were digitized with a NanoZoomer HT scan system (Hamamatsu Photonics, Japan). The densities of TILs were assessed in accordance with a previous report with some modification [12]. Briefly, $0.30 \mathrm{~mm}^{2}$ of the representative tumor area in each core was selected, and the number of positive cells was counted in a total area of $0.60 \mathrm{~mm}^{2}$. The median values were used for cutoff points for high densities of TILs (vs low densities of TILs).

\section{Evaluation of MMR status}

Tumors were considered negative for MLH1, MSH2, PMS2, or MSH6 expression only if there was a complete absence of nuclear staining in the TCs, and normal epithelial cells and lymphocytes were used as an internal control. Tumors lacking MLH1, MSH2, PMS2, or MSH6 expression were considered to be MMR deficient, whereas tumors that maintained expression of all markers were considered to be MMR proficient.

\section{EBV ISH}

Chromogenic ISH for EBV-encoded RNA (EBER) was performed with fluorescein-labeled oligonucleotide probes (INFORM EBER probe, Ventana) with enzymatic digestion (ISH protease 3, Ventana) and an iViewBlue detection kit (Ventana) with use of the BenchMark ULTRA staining system.

\section{Statistical analysis}

Comparisons of categorical variables were done by a chisquare test or Fisher's exact test as appropriate. We

\section{Panel 1}
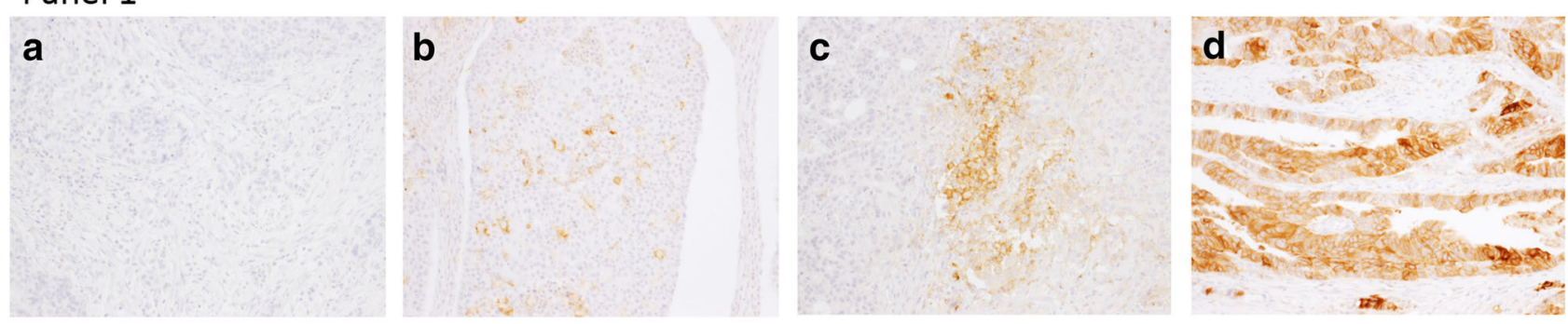

\section{Panel 2}

e

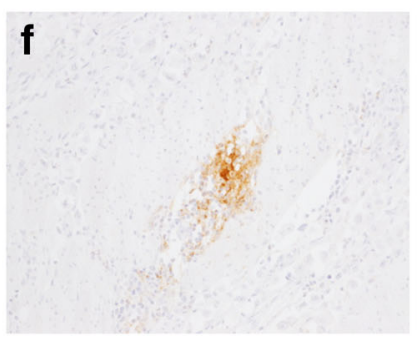

Fig. 1 Representative images of programmed death ligand 1 expression on tumor cells (panel 1) and tumor-infiltrating immune cells (panel 2): a immunohistochemistry (IHC) score 0; b IHC score 1+;
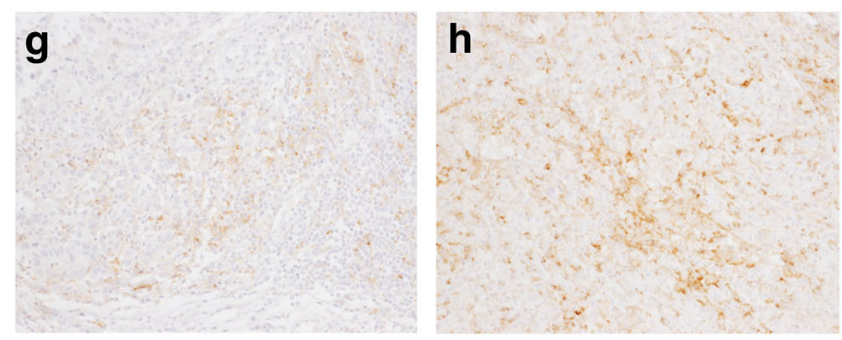

c IHC score 2+; d IHC score 3+; e IHC score 0; f IHC score 1+; g IHC score 2+; h IHC score $3+$ 
performed survival analyses in the patients with R0 resection. OS was defined as the time from the date of surgery until death from any cause. Patients who were alive were censored at the last follow-up date. OS rates were estimated by the Kaplan-Meier method, and differences between the groups according to PD-L1 expression, densities of TILs, MMR status, and EBV status were identified by univariate and multivariate analyzes by Cox proportional hazards models and are presented as hazard ratios with $95 \%$ confidence intervals. Confounders in univariate and multivariate analyses included age, histological type, depth of invasion, lymphatic invasion, venous invasion, TNM stage, and adjuvant chemotherapy. Statistical analyses were performed with IBM SPSS Statistics version 21 (IBM, Armonk, NY, USA). All tests were two-sided, and differences were considered significant when $P<0.05$.

\section{Results}

\section{Prevalence of PD-L1 expression according to MMR and EBV status}

The PD-L1 IHC scores for the TCs were 0 in 376 patients $(77.2 \%), 1+$ in 67 patients $(13.8 \%), 2+$ in 23 patients $(4.7 \%)$, and $3+$ in 21 patients $(4.3 \%)$ (Table 1). The scores for the TIICs were 0 in 188 patients $(38.6 \%), 1+$ in 278 patients $(57.1 \%), 2+$ in 19 patients $(3.9 \%)$, and $3+$ in 2 patients $(0.4 \%)$. As a result, PD-L1 expression on TCs and TIICs (IHC scores $1+, 2+$, and $3+$ ) was positive in 22.8 and $61.4 \%$ of all cases respectively. Most of the patients who were PD-L1 positive for the TCs demonstrated PD-L1 positivity on TIICs as well, leading to the observation that $61.8 \%$ of patients had positive PD-L1 expression on either TCs or TIICs. The concordance rate of PD-L1 IHC scores on TCs between the two TMA cores was $83.2 \%$, whereas that on TIICs was $65.3 \%$ (Table S1).

MMR deficiency was observed in 25 cases (5.1\%), and PD-L1 positivity was more frequent in the event of MMR deficiency than in the event of MMR proficiency on both TCs $(72.0 \%$ vs $20.1 \%, P<0.001)$ and TIICs $(96.0 \%$ vs $59.5 \%, P<0.001$ ) (Table 1, Fig. 2). A PD-L1 IHC score of $2+/ 3+$ was significantly more frequent in MMR deficiency cases than in MMR proficiency cases (TCs $36.0 \%$ vs $7.6 \%, P<0.001$; TIICs $24.0 \%$ vs $3.2 \% ; P<0.001$ ). The expression status of each MMR protein is presented in Table S2.

EBV was detected in 25 patients (5.1\%), and PD-L1 positivity on TCs was more frequent in EBV-positive GC than in EBV-negative GC (52.0\% vs $21.2 \%, P=0.001)$ (Table 1, Fig. 2). A PD-L1 IHC score of $2+/ 3+$ on TCs was also more frequently observed in EBV-positive GG ( $24.0 \%$ vs $8.2 \%, P=0.022)$. PD-L1 positivity on TIICs was also more frequent in EBV-positive GG $(80.0 \%$ vs $60.4 \%, P<0.001)$.

\section{Clinicopathological features associated with PD-L1 expression}

Baseline patient characteristics are presented in Table 2. The median age was 66 years (range 26-92 years), and there were 327 male patients $(67.1 \%)$. Cancer stage

Table 1 Prevalence of programmed death ligand $1(P D-L 1)$ expression according to mismatch repair $(M M R)$ status and Epstein-Barr virus $(E B V)$ status

\begin{tabular}{|c|c|c|c|c|c|c|c|}
\hline & $\begin{array}{l}\text { All } \\
(N=487)\end{array}$ & $\begin{array}{l}\text { P-MMR } \\
(n=462)\end{array}$ & $\begin{array}{l}\text { D-MMR } \\
(n=25)\end{array}$ & $P$ & $\begin{array}{l}\text { EBV negative } \\
(n=462)\end{array}$ & $\begin{array}{l}\text { EBV positive } \\
(n=25)\end{array}$ & $P$ \\
\hline \multicolumn{8}{|l|}{ TCs } \\
\hline $\begin{array}{l}\text { No PD-L1 expression in } \\
\text { TCs }\end{array}$ & $376(77.2 \%)$ & $369(79.9 \%)$ & $7(28.0 \%)$ & $<0.001$ & $364(78.8 \%)$ & $12(48.0 \%)$ & 0.001 \\
\hline PD-L1 expression in TCs & $111(22.8 \%)$ & $93(20.1 \%)$ & $18(72.0 \%)$ & & $98(21.2 \%)$ & $13(52.0 \%)$ & \\
\hline IHC score $1+$ & $67(13.8 \%)$ & $58(12.5 \%)$ & $9(36.0 \%)$ & $<0.001$ & $60(13.0 \%)$ & $7(28.0 \%)$ & 0.022 \\
\hline IHC score $2+$ & $23(4.7 \%)$ & $19(4.1 \%)$ & $4(16.0 \%)$ & & $20(4.3 \%)$ & $3(12.0 \%)$ & \\
\hline IHC score $3+$ & $21(4.3 \%)$ & $16(3.5 \%)$ & $5(20.0 \%)$ & & $18(3.9 \%)$ & $3(12.0 \%)$ & \\
\hline \multicolumn{8}{|l|}{ TIICs } \\
\hline $\begin{array}{l}\text { No PD-L1 expression in } \\
\text { TIICs }\end{array}$ & $188(38.6 \%)$ & $187(40.5 \%)$ & $1(4.0 \%)$ & $<0.001$ & $183(39.6 \%)$ & $5(20.0 \%)$ & 0.050 \\
\hline $\begin{array}{l}\text { PD-L1 expression in } \\
\text { TIICs }\end{array}$ & $299(61.4 \%)$ & $275(59.5 \%)$ & $24(96.0 \%)$ & & $279(60.4 \%)$ & $20(80.0 \%)$ & \\
\hline IHC score $1+$ & $278(57.1 \%)$ & $260(56.3 \%)$ & $18(72.0 \%)$ & $<0.001$ & $259(56.1 \%)$ & $19(76.0 \%)$ & 1.00 \\
\hline IHC score $2+$ & $19(3.9 \%)$ & $14(3.0 \%)$ & $5(20.0 \%)$ & & $18(3.9 \%)$ & $1(4.0 \%)$ & \\
\hline IHC score $3+$ & $2(0.4 \%)$ & $1(0.2 \%)$ & $1(4.0 \%)$ & & $2(0.4 \%)$ & $0(0 \%)$ & \\
\hline
\end{tabular}

D-MMR MMR deficient, IHC immunohistochemistry, P-MMR MMR proficient, TCs tumor cells, TIICs tumor-infiltrating immune cells 
Panel 1

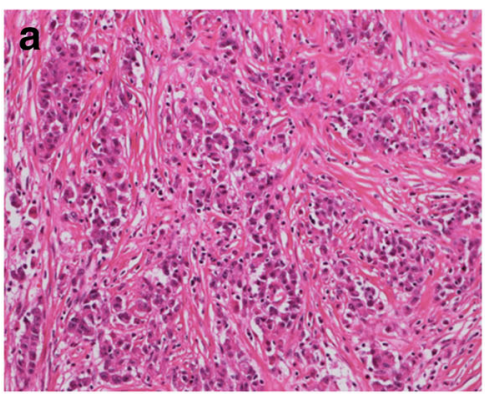

Panel 2

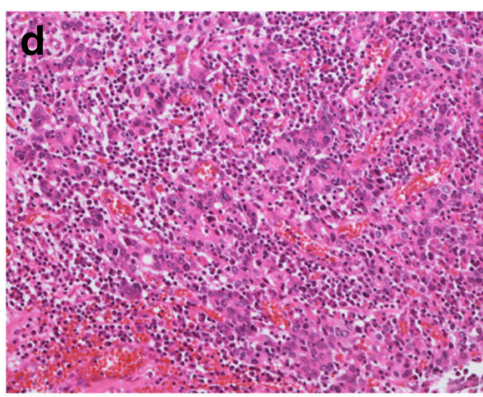

b
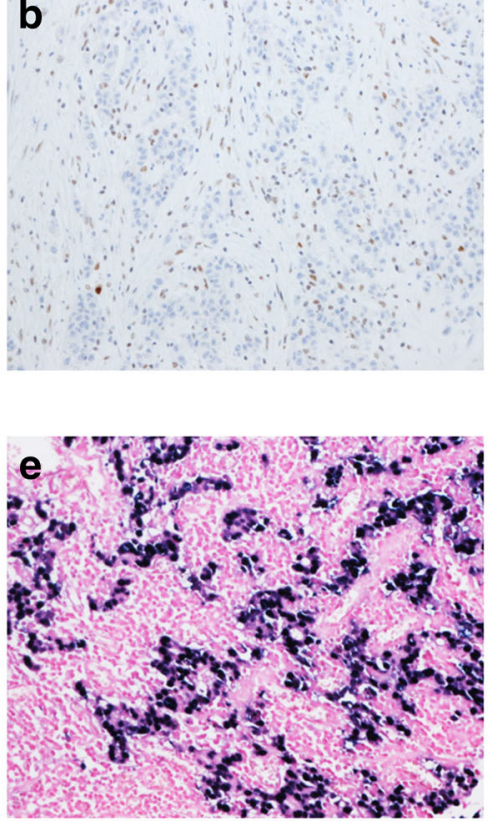
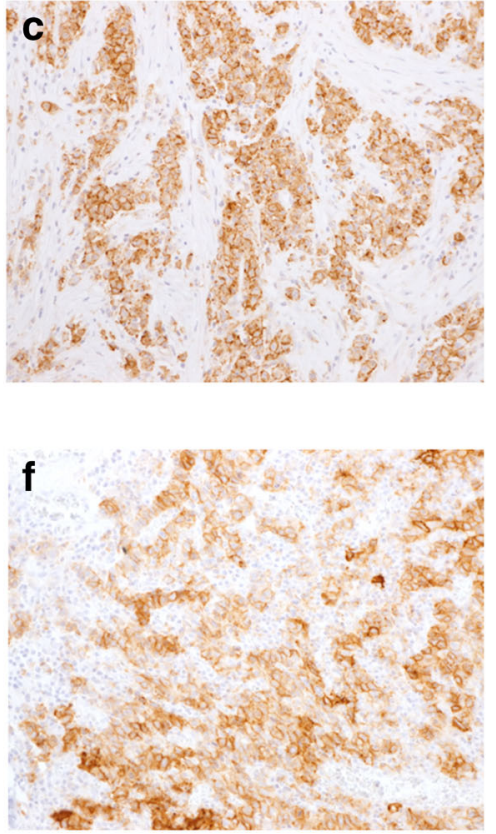

Fig. 2 Prevalence of programmed death ligand 1 (PD-L1) expression in mismatch repair (MMR)-deficient and Epstein-Barr virus (EBV)positive gastric cancer. PD-L1 expression was more frequently observed in MMR-deficient and EBV-positive gastric cancer. Representative images of a case with MMR deficiency (panel 1) and EBV

included 358 cases of stage III GC (73.5\%) and 129 cases of stage IV GC (26.5\%). R0 resection and adjuvant chemotherapy were performed in 383 patients $(78.6 \%)$ and 261 patients $(53.6 \%)$ respectively.

PD-L1 expression was more frequently observed in patients aged 65 years or older than in those younger than 65 years (TCs $28.1 \%$ vs $16.4 \%, P=0.002$ ), in males than in females (TCs $25.7 \%$ vs $16.9 \%, P=0.029$; TIICs $64.5 \%$ vs $55.0 \%, P=0.043$ ), and in poorly differentiated adenocarcinoma with solid-type histological features than in other histological subtypes (TCs $P<0.001$; TIICs $P<0.001$ ) (Table 2).

\section{Associations between PD-L1 expression and TILs}

The median densities of $\mathrm{CD}^{+}, \mathrm{CD}^{+}, \mathrm{CD}^{+}$, and $\mathrm{FOXP}^{+}$ TILs were $725 / \mathrm{mm}^{2}, 303 / \mathrm{mm}^{2}, 384 / \mathrm{mm}^{2}$, and $53 / \mathrm{mm}^{2}$ respectively. As shown in Table 3 and Fig. 3, 78 \% of the PDL1-positive TC tumors were associated with high densities of $\mathrm{CD}^{+}$TILs, whereas $42 \%$ of PD-L1-negative tumors were $(P<0.001)$. Moreover, $65 \%$ of PD-L1-positive TIIC tumors were associated with high densities of $\mathrm{CD} 8^{+}$TILs, whereas $27 \%$ of PD-L1-negative tumors were $(P<0.001)$. A strong association was also observed between PD-L1 expression and high densities of $\mathrm{CD}^{+}$and FOXP3 ${ }^{+}$TILs (TCs $P<0.00$; TIICs $P<0.001)$. In addition, there was association between positivity (panel 2) with PD-L1 immunohistochemistry (IHC) score $3+$ on tumor cells: a hematoxylin and eosin staining; b mutL homolog 1 loss; c PD-L1 IHC score 3+; d hematoxylin and eosin staining; e EBV positivity; f PD-L1 IHC score 3+

PD-L1 expression and high densities of $\mathrm{CD} 4^{+}$TILs (TCs $P=0.004$; TIICs $P=0.008$ ), although it was not as strong as that of $\mathrm{CD}^{+}, \mathrm{CD}^{+}$, and $\mathrm{FOXP3}^{+}$TILs.

\section{Survival analysis}

The survival analyses of the 383 patients with R0 resection are presented in Fig. 4. In the multivariate analysis, high densities of $\mathrm{CD}^{+}$TILs were significantly associated with better survival (hazard ratio 0.63; $95 \%$ confidence interval 0.39-0.99; $P=0.050$ ), whereas PD-L1 expression on TCs and TIICs, the densities of other subtypes of TILs, MMR status, and EBV status were not independent prognostic factors (Table S3).

\section{Discussion}

In this study, we investigated PD-L1 expression levels on the surface of TCs and TIICs with TIL, MMR, and EBV status in 487 surgically resected specimens of GC. We found a higher PD-L1 positivity in MMR-deficient and EBV-positive GC. Furthermore, we elucidated a strong association between PD-L1 expression and high densities of TILs. The other clinicopathological features related to a higher PD-L1 positivity were old age, male sex, and poorly 
Table 2 Patient characteristics according to programmed death ligand $1(P D-L 1)$ expression

\begin{tabular}{|c|c|c|c|c|c|c|c|}
\hline & All $(N=487)$ & TCs $(-)(n=376)$ & $\operatorname{TCs}(+)(n=111)$ & $P$ & TIICs $(-)(n=188)$ & TIICs $(+)(n=299)$ & $P$ \\
\hline \multicolumn{8}{|l|}{ Age (years) ${ }^{\mathrm{a}}$} \\
\hline$<65$ & $220(45.2 \%)$ & $184(48.9 \%)$ & $36(32.4 \%)$ & \multirow[t]{2}{*}{0.002} & $95(50.5 \%)$ & $125(41.8 \%)$ & \multirow[t]{2}{*}{0.060} \\
\hline$\geq 65$ & $267(54.8 \%)$ & $192(51.1 \%)$ & $75(67.6 \%)$ & & $93(49.5 \%)$ & $174(58.2 \%)$ & \\
\hline \multicolumn{8}{|l|}{ Sex } \\
\hline Male & $327(67.1 \%)$ & $243(64.6 \%)$ & $84(75.7 \%)$ & \multirow[t]{2}{*}{0.029} & $116(61.7 \%)$ & $211(70.6 \%)$ & \multirow[t]{2}{*}{0.043} \\
\hline Female & $160(32.9 \%)$ & $133(35.4 \%)$ & $27(24.3 \%)$ & & $72(38.3 \%)$ & $88(29.4 \%)$ & \\
\hline \multicolumn{8}{|l|}{ Tumor location } \\
\hline EGJ & $33(6.8 \%)$ & $26(6.9 \%)$ & $7(6.3 \%)$ & \multirow[t]{5}{*}{0.737} & $17(9.0 \%)$ & $16(5.4 \%)$ & \multirow[t]{5}{*}{0.220} \\
\hline Upper third & $103(21.1 \%)$ & $80(21.3 \%)$ & $23(20.7 \%)$ & & $41(21.8 \%)$ & $62(20.7 \%)$ & \\
\hline Middle third & $195(40.1 \%)$ & $153(40.6 \%)$ & $42(37.8 \%)$ & & $80(42.6 \%)$ & $115(38.5 \%)$ & \\
\hline Lower third & $149(30.6 \%)$ & $113(30.1 \%)$ & $36(32.5 \%)$ & & $48(25.5 \%)$ & $101(33.8 \%)$ & \\
\hline Remnant & $7(1.4 \%)$ & $4(1.1 \%)$ & $3(2.7 \%)$ & & $2(1.1 \%)$ & $5(1.7 \%)$ & \\
\hline \multicolumn{8}{|c|}{ Histological type ${ }^{b}$} \\
\hline pap & $15(3.1 \%)$ & $12(3.2 \%)$ & $3(2.7 \%)$ & \multirow[t]{7}{*}{$<0.001$} & $7(3.7 \%)$ & $8(2.7 \%)$ & \multirow[t]{7}{*}{$<0.001$} \\
\hline tub1 & $18(3.7 \%)$ & $16(4.3 \%)$ & $2(1.8 \%)$ & & $11(5.9 \%)$ & $7(2.3 \%)$ & \\
\hline tub2 & $151(31.0 \%)$ & $114(30.3 \%)$ & $37(33.3 \%)$ & & $44(23.4 \%)$ & $107(35.8 \%)$ & \\
\hline por1 & $46(9.4 \%)$ & $22(5.9 \%)$ & $24(21.6 \%)$ & & $5(2.7 \%)$ & $41(13.7 \%)$ & \\
\hline por2 & $214(43.9 \%)$ & $170(45.1 \%)$ & $44(39.6 \%)$ & & $92(48.9 \%)$ & $122(40.8 \%)$ & \\
\hline $\operatorname{sig}$ & $13(2.7 \%)$ & $13(3.5 \%)$ & $0(0 \%)$ & & $5(2.7 \%)$ & $8(2.7 \%)$ & \\
\hline muc & $30(6.2 \%)$ & $29(7.7 \%)$ & $1(0.9 \%)$ & & $24(12.8 \%)$ & $6(2.0 \%)$ & \\
\hline \multicolumn{8}{|c|}{ Lymphatic invasion } \\
\hline Absent & $38(7.8 \%)$ & $26(6.9 \%)$ & $12(10.8 \%)$ & \multirow[t]{2}{*}{0.179} & $12(6.4 \%)$ & $26(8.7 \%)$ & \multirow[t]{2}{*}{0.354} \\
\hline Present & $449(92.2 \%)$ & $350(93.1 \%)$ & $99(89.2 \%)$ & & $176(93.6 \%)$ & $273(91.3 \%)$ & \\
\hline \multicolumn{8}{|c|}{ Venous invasion } \\
\hline Absent & $55(11.3 \%)$ & $48(12.8 \%)$ & $7(6.3 \%)$ & \multirow[t]{2}{*}{0.059} & $20(10.6 \%)$ & $35(11.7 \%)$ & \multirow[t]{2}{*}{0.717} \\
\hline Present & $432(88.7 \%)$ & $328(87.2 \%)$ & $104(93.7 \%)$ & & $168(89.4 \%)$ & $264(88.3 \%)$ & \\
\hline \multicolumn{8}{|c|}{ Depth of invasion } \\
\hline $\mathrm{T} 1$ & $1(0.2 \%)$ & $1(0.3 \%)$ & $0(0 \%)$ & \multirow[t]{4}{*}{0.635} & $0(0 \%)$ & $1(0.3 \%)$ & \multirow[t]{4}{*}{0.143} \\
\hline $\mathrm{T} 2$ & $21(4.3 \%)$ & $14(3.7 \%)$ & $7(6.3 \%)$ & & $5(2.7 \%)$ & $16(5.4 \%)$ & \\
\hline $\mathrm{T} 3$ & $166(34.1 \%)$ & $128(34.0 \%)$ & $38(34.2 \%)$ & & $57(30.3 \%)$ & $109(36.5 \%)$ & \\
\hline $\mathrm{T} 4$ & $299(61.4 \%)$ & $233(62.0)$ & $66(59.5)$ & & $126(67.0 \%)$ & $173(57.9 \%)$ & \\
\hline \multicolumn{8}{|c|}{ Regional lymph node invasion } \\
\hline Absent & $7(1.4 \%)$ & $4(1.1)$ & $3(2.7 \%)$ & \multirow[t]{2}{*}{0.202} & $0(0 \%)$ & $7(2.3 \%)$ & 0.035 \\
\hline Present & $480(98.6 \%)$ & $372(98.9 \%)$ & $108(97.3 \%)$ & & $188(100 \%)$ & $292(97.7 \%)$ & \\
\hline TNM stage & & & & & & & \\
\hline III & $358(73.5 \%)$ & $274(72.9 \%)$ & $84(75.7 \%)$ & 0.556 & $138(73.4 \%)$ & $220(73.6 \%)$ & 0.966 \\
\hline IV & $129(26.5 \%)$ & $102(27.1 \%)$ & $27(24.3 \%)$ & & $50(26.6 \%)$ & $79(26.4 \%)$ & \\
\hline Residual tumor & & & & & & & \\
\hline R0 & $383(78.6 \%)$ & - & - & & - & - & \\
\hline $\mathrm{R} 1, \mathrm{R} 2$ & $104(21.4 \%)$ & - & - & & - & - & \\
\hline Adjuvant chem & otherapy & & & & & & \\
\hline Yes & $261(53.6 \%)$ & - & - & & - & - & \\
\hline No & $226(46.4 \%)$ & - & - & & - & - & \\
\hline
\end{tabular}

EGJ esophagogastric junction, muc mucinous adenocarcinoma, pap papillary adenocarcinoma, porl solid-type poorly differentiated adenocarcinoma, por 2 non-solid-type poorly differentiated adenocarcinoma, sig signet ring cell carcinoma, TCs tumor cells, TIICs tumor-infiltrating immune cells, $t u b 1$ well-differentiated tubular adenocarcinoma, tub2 moderately differentiated tubular adenocarcinoma

${ }^{a}$ Median 66 years, range 26-92 years

${ }^{\mathrm{b}}$ Histological classification is according to the Japanese classification of gastric carcinoma (third English edition) 
Table 3 Association between programmed death ligand $1(P D-L 1)$ expression and tumor-infiltrating lymphocytes

\begin{tabular}{|c|c|c|c|c|c|c|c|c|}
\hline & \multicolumn{2}{|l|}{ CD3 } & \multicolumn{2}{|l|}{ CD4 } & \multicolumn{2}{|l|}{$\mathrm{CD} 8$} & \multicolumn{2}{|l|}{ FOXP3 } \\
\hline & High & Low & High & Low & High & Low & High & Low \\
\hline \multicolumn{9}{|c|}{ PD-L1 in TCs } \\
\hline Positive & $86(77 \%)$ & $25(23 \%)$ & $69(62 \%)$ & $42(38 \%)$ & $87(78 \%)$ & $24(22 \%)$ & $84(76 \%)$ & $27(24 \%)$ \\
\hline Negative & $157(42 \%)$ & $219(58 \%)$ & $175(47 \%)$ & $201(53 \%)$ & $157(42 \%)$ & $219(58 \%)$ & $158(42 \%)$ & $218(58 \%)$ \\
\hline$P$ & $<0.001$ & & 0.004 & & $<0.001$ & & $<0.001$ & \\
\hline \multicolumn{9}{|c|}{ PD-L1 in TIICs } \\
\hline Positive & $188(63 \%)$ & $111(37 \%)$ & $164(55 \%)$ & $135(45 \%)$ & $193(65 \%)$ & $106(35 \%)$ & $179(60 \%)$ & $120(40 \%)$ \\
\hline Negative & $55(29 \%)$ & $133(71 \%)$ & $80(43 \%)$ & $108(57 \%)$ & $51(27 \%)$ & $137(73 \%)$ & $63(33 \%)$ & $125(67 \%)$ \\
\hline$P$ & $<0.001$ & & 0.008 & & $<0.001$ & & $<0.001$ & \\
\hline
\end{tabular}

The median densities of tumor-infiltrating lymphocytes positive for CD3, CD4, CD8, and forkhead box P3 (FOXP3) were $725 / \mathrm{mm}^{2}, 303 / \mathrm{mm}^{2}$, $384 / \mathrm{mm}^{2}$, and $53 / \mathrm{mm}^{2}$ respectively

TCs tumor cells, TIICs tumor-infiltrating immune cells
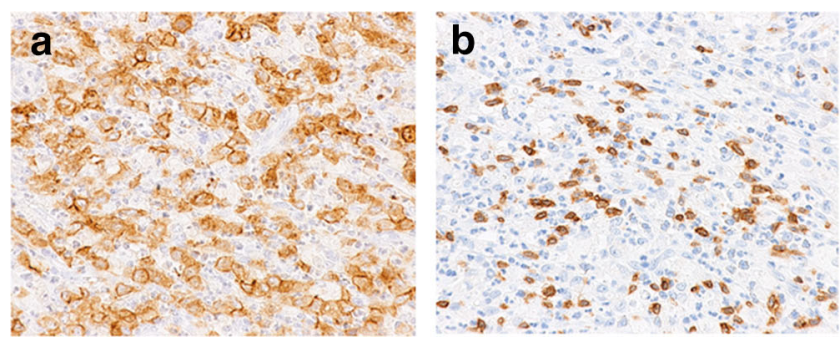

Fig. 3 A strong association was observed between programmed death ligand 1 (PD-L1) expression and high densities of $\mathrm{CD}^{+}$, $\mathrm{CD}^{+}$, and forkhead box P3 (FOXP3)-positive tumor-infiltrating

differentiated adenocarcinoma with solid-type histological features. To our knowledge, this study is the first to provide information in a large cohort of GC patients, including the association of GC with clinicopathological comprehensive features of PD-L1 expression with TIL, MMR, and EBV status.

In our patient cohort, $61.8 \%$ of patients exhibited positive PD-L1 expression on either TCs or TIICs, comparable to the rate seen in the phase I trial of pembrolizumab for advanced GC [6]. In the trial, a trend toward an association between higher levels of PD-L1 expression on TCs or TIICs and an objective response rate, progression-free survival, and OS was observed.

Importantly, the concordance rate of PD-L1 IHC scores on TCs between two TMA cores was $83.2 \%$, whereas that on TIICs was $65.3 \%$ in our analysis. It has recently been reported that PD-L1 expression in non-small-cell lung cancer was frequently discordant between surgically resected and matched biopsy specimens (the overall discordance rate was $48 \%$ ), primarily due to the lack of PDL1-positive TIIC components in matched biopsies [13]. Our study also showed that the concordance rate of PD-L1 IHC scores on TIICs was relatively lower than that on TCs
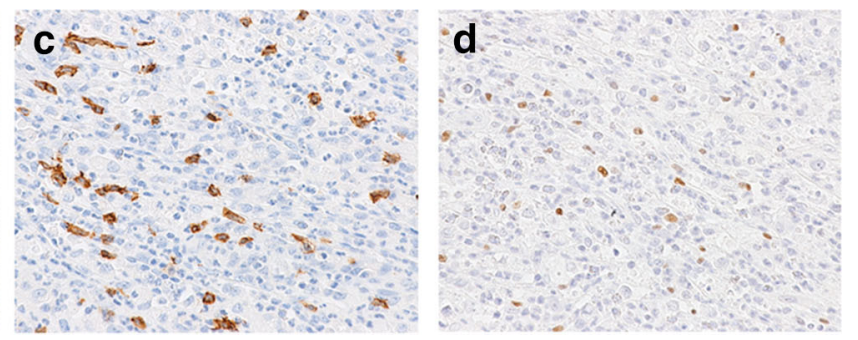

lymphocytes (TILs). Representative images of a case with a PD-L1 immunohistochemistry score $3+$ on tumor cells and high densities of b $\mathrm{CD}^{+}, \mathbf{c ~ C D}^{+}$, and $\mathbf{d ~ F O X P} 3^{+}$TILs

in GC. To avoid underestimation of PD-L1-positive tumors in GC, it might be important for the IHC analysis to be performed on multiple biopsy specimens.

Recently, pembrolizumab has shown promising efficacy in a phase II trial for patients with MMR-deficient tumors [10]. It was shown that MMR-deficient colorectal cancers had higher mutation loads compared with MMR-proficient colorectal cancers, leading to high infiltration of $\mathrm{CD} 8^{+} \mathrm{T}$ cells, presumably due to recognition of a large number of tumor neoantigens and the corresponding expression of immune-checkpoint-related molecules in the tumor microenvironment [14]. Our study also found that PD-L1 positivity on TCs and TIICs was significantly higher in MMR-deficient GC cases. Moreover, $72 \%$ of cases of MMR deficiency exhibited high densities of $\mathrm{CD}^{+}$TILs (data not shown).

The Cancer Genome Atlas Research Network [9] revealed that PD-L1 gene amplification was elevated in EBV-positive GC. In our analysis, PD-L1 protein expression was more frequently observed, not only on TCs but also on TIICs, in EBV-positive GC than in EBV-negative GC. Furthermore, a high rate of infiltration of $\mathrm{CD}^{+} \mathrm{T}$ cells is one of the characteristic features of EBV-positive GC 

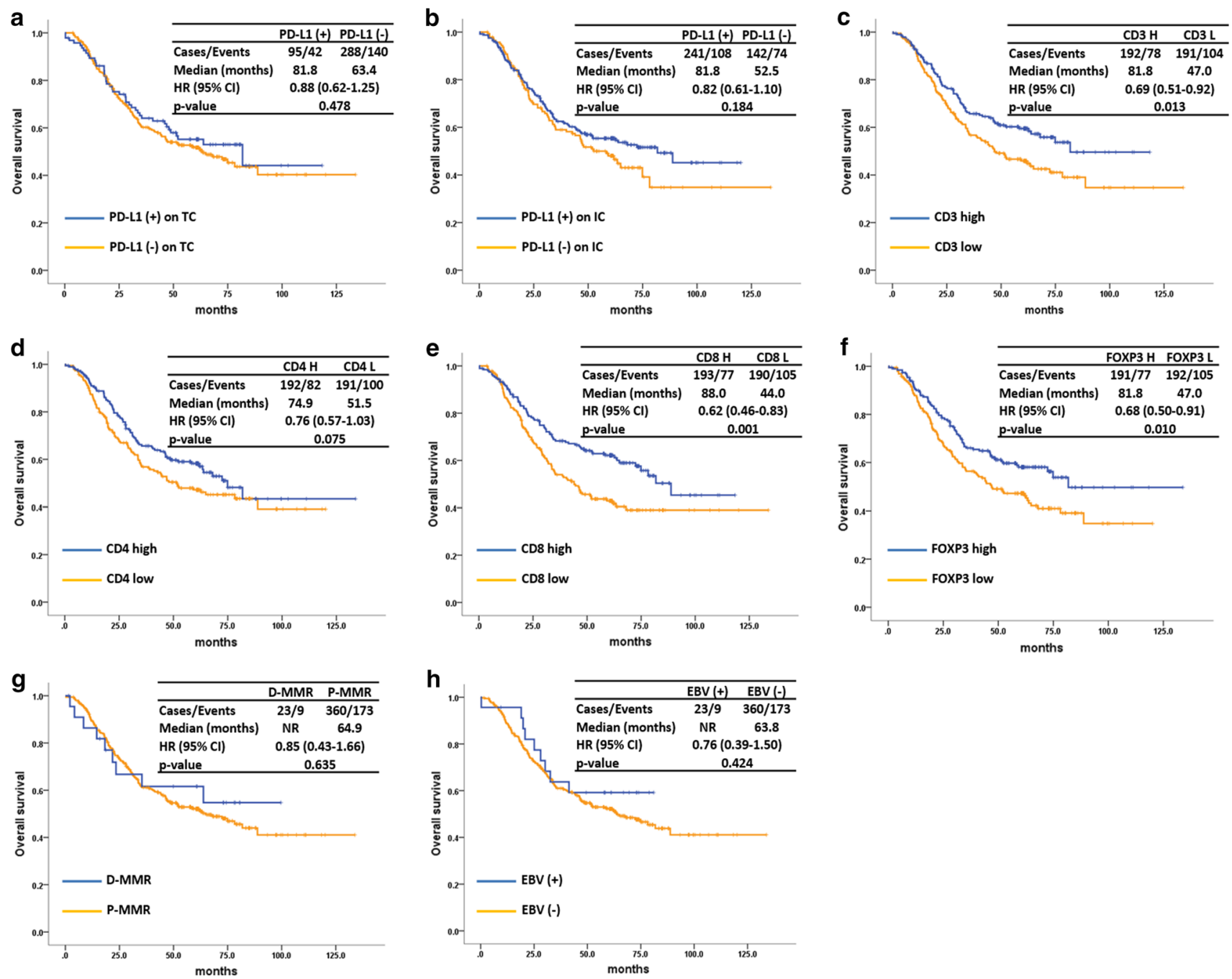

Fig. 4 Kaplan-Meier plots of overall survival according to programmed death ligand $1(P D-L 1)$ expression, densities of tumorinfiltrating lymphocytes, mismatch repair (MMR) status, and EpsteinBarr virus $(E B V)$ status: a PD-L1 positive versus PD-L1 negative on tumor cells $(T C)$; b PD-L1 positive versus PD-L1 negative on tumorinfiltrating immune cells (TIIC); $\mathbf{c}$ high density versus low density of

[15]. Indeed, 24 of 25 cases (96\%) of EBV-positive GC in this study were associated with high densities of $\mathrm{CD}^{+}$ TILs (data not shown). A recent study showed that the clinical efficacy of pembrolizumab for melanoma was correlated with increased frequencies of preexisting $\mathrm{CD} 8^{+}$ $\mathrm{T}$ cells [9]. Considering these findings, anti-PD-1/PD-L1 antibodies might have more therapeutic efficacy on EBVpositive GC.

It has been considered that there are two general mechanisms for the expression of PD-L1 on TCs: (1) innate immune resistance and (2) adaptive immune resistance [5]. In terms of innate immune resistance, constitutive oncogenic signaling induces PD-L1 expression in TCs, whereas in cases of adaptive immune resistance, tumorinfiltrating $\mathrm{T}$ cells induce PD-L1 upregulation on TCs, as
$\mathrm{CD}^{+}$tumor-infiltrating lymphocytes (TILs); d high density versus low density of $\mathrm{CD} 4^{+}$TILs; e high density versus low density of $\mathrm{CD}^{+}$ TILs; $\mathbf{f}$ high density versus low density of forkhead box P3 (FOXP3)positive TILs; $\mathbf{g}$ MMR deficient $(D-M M R)$ versus MMR proficient $(P$ $M M R)$; h EBV positive versus EBV negative. $C I$ confidence interval, $H$ high, $H R$ hazard ratio, $L$ low

shown in a previous report on melanoma [16]. Our study demonstrated that approximately $80 \%$ of PD-L1-positive TC cases were associated with high densities of $\mathrm{CD}^{+}$and $\mathrm{CD}^{+}$TILs. This may suggest that an adaptive immune resistance contributes more to PD-L1 expression in GC than innate immune resistance. We found that PD-L1 expression was also strongly associated with high densities of FOXP $3^{+}$TILs, a representative marker of regulatory $\mathrm{T}$ cells, which are known to suppress the activity of cytotoxic $\mathrm{T}$ cells. Although we did not analyze the function of regulatory $\mathrm{T}$ cells in this study, targeting regulatory $\mathrm{T}$ cells might also enhance the activity of anti-PD-1/PD-L1 antibodies in GC.

The impact of PD-L1 expression on prognosis remains controversial in several malignancies [16-20]. In GC, one 
report found that high PD-L1 expression in tumors was associated with a poor prognosis [17], whereas another showed an improved prognosis [18]. In our study, no association between PD-L1 expression and the prognosis was observed. This variability in reported outcomes might be influenced in part by the patient cohort (clinical stages) examined and the evaluation criteria for PD-L1 expression.

A major limitation of our study is that we investigated only a small portion of the total tumor volume, since we used TMAs.

In conclusion, PD-L1 expression in GC had distinct clinicopathological features, including high densities of TILs, MMR deficiency, and EBV positivity. We also showed that PD-L1 expression was not a prognostic factor in GC. The impact of these characteristics on the efficacy of anti-PD-1/PD-L1 antibodies warrants further evaluation.

\section{Compliance with ethical standards}

Conflict of interest The authors declare that they have no conflict of interest.

Human rights statement and informed consent All procedures followed were in accordance with the ethical standards of the responsible committee on human experimentation (institutional and national) and with the Helsinki Declaration of 1964 and later versions. Informed consent or substitute for it was obtained from all patients for their being included in the study.

\section{References}

1. Topalian SL, Hodi FS, Brahmer JR, Gettinger SN, Smith DC, McDermott DF, et al. Safety, activity, and immune correlates of anti-PD-1 antibody in cancer. $\mathrm{N}$ Engl $\mathrm{J}$ Med. 2012;366(26):2443-54.

2. Robert C, Long GV, Brady B, Dutriaux C, Maio M, Mortier L, et al. Nivolumab in previously untreated melanoma without BRAF mutation. N Engl J Med. 2015;372(4):320-30.

3. Garon EB, Rizvi NA, Hui R, Leighl N, Balmanoukian AS, Eder JP, et al. Pembrolizumab for the treatment of non-small-cell lung cancer. N Engl J Med. 2015;372(21):2018-28.

4. Motzer RJ, Escudier B, McDermott DF, George S, Hammers HJ, Srinivas $\mathrm{S}$, et al. Nivolumab versus everolimus in advanced renalcell carcinoma. N Engl J Med. 2015;373(19):1803-13.

5. Pardoll DM. The blockade of immune checkpoints in cancer immunotherapy. Nat Rev Cancer. 2012;12(4):252-64.

6. Muro K, Bang YJ, Shankaran V, Geva R, Catenacci D-VT, Gupta $\mathrm{S}$, et al. Relationship between PD-L1 expression and clinical outcomes in patients (pts) with advanced gastric cancer treated with the anti-PD-1 monoclonal antibody pembrolizumab (Pembro; MK-3475) in KEYNOTE-012. J Clin Oncol. 2015;33(3 Suppl):3.
7. Horn L, Spigel DR, Gettinger SN, Antonia SJ, Gordon MS, Herbst RS, et al. Clinical activity, safety and predictive biomarkers of the engineered antibody MPDL3280A (anti-PDL1) in non-small cell lung cancer (NSCLC): update from a phase Ia study. J Clin Oncol. 2015;33(15 Suppl):8029.

8. Tumeh PC, Harview CL, Yearley JH, Shintaku IP, Taylor EJ, Robert L, et al. PD-1 blockade induces responses by inhibiting adaptive immune resistance. Nature. 2014;515(7528):568-71.

9. Cancer Genome Atlas Research Network. Comprehensive molecular characterization of gastric adenocarcinoma. Nature. 2014;513(7517):202-9.

10. Le DT, Uram JN, Wang H, Bartlett BR, Kemberling H, Eyring $\mathrm{AD}$, et al. PD-1 blockade in tumors with mismatch-repair deficiency. N Engl J Med. 2015;372(26):2509-20.

11. Kuboki Y, Yamashita S, Niwa T, Ushijima T, Nagatsuma A, Kuwata $\mathrm{T}$, et al. Comprehensive analyses using next-generation sequencing and immunohistochemistry enable precise treatment in advanced gastric cancer. Ann Oncol. 2016;27(1):127-33.

12. Lee HE, Chae SW, Lee YJ, Kim MA, Lee HS, Lee BL, et al. Prognostic implications of type and density of tumour-infiltrating lymphocytes in gastric cancer. $\mathrm{Br} \mathrm{J}$ Cancer. 2008;99(10): 1704-11.

13. Ilie M, Long-Mira E, Bence C, Butori C, Lassalle S, Bouhlel L, et al. Comparative study of the PD-L1 status between surgically resected specimens and matched biopsies of NSCLC patients reveal major discordances: a potential issue for anti-PD-L1 therapeutic strategies. Ann Oncol. 2016;27(1):147-53.

14. Llosa NJ, Cruise M, Tam A, Wicks EC, Hechenbleikner EM, Taube JM, et al. The vigorous immune microenvironment of microsatellite instable colon cancer is balanced by multiple counter-inhibitory checkpoints. Cancer Discov. 2015;5(1):43-51.

15. Shinozaki-Ushiku A, Kunita A, Fukayama M. Update on Epstein-Barr virus and gastric cancer (review). Int $\mathrm{J}$ Oncol. 2015;46(4):1421-34.

16. Taube JM, Anders RA, Young GD, Xu H, Sharma R, McMiller $\mathrm{TL}$, et al. Colocalization of inflammatory response with B7-h1 expression in human melanocytic lesions supports an adaptive resistance mechanism of immune escape. Sci Transl Med. 2012;4(127):127ra37.

17. Eto S, Yoshikawa K, Nishi M, Higashijima J, Tokunaga T, Nakao $\mathrm{T}$, et al. Programmed cell death protein 1 expression is an independent prognostic factor in gastric cancer after curative resection. Gastric Cancer. 2015;19(2):466-71.

18. Kim JW, Nam KH, Ahn SH, Park do J, Kim HH, Kim SH, et al. Prognostic implications of immunosuppressive protein expression in tumors as well as immune cell infiltration within the tumor microenvironment in gastric cancer. Gastric Cancer. 2014;19(1):42-52.

19. Hamanishi J, Mandai M, Iwasaki M, Okazaki T, Tanaka Y, Yamaguchi K, et al. Programmed cell death 1 ligand 1 and tumorinfiltrating CD8 $+\mathrm{T}$ lymphocytes are prognostic factors of human ovarian cancer. Proc Natl Acad Sci U S A. 2007;104(9):3360-5.

20. Yang CY, Lin MW, Chang YL, Wu CT, Yang PC. Programmed cell death-ligand 1 expression is associated with a favourable immune microenvironment and better overall survival in stage I pulmonary squamous cell carcinoma. Eur $\mathrm{J}$ Cancer. 2016;57:91-103. 